\title{
Prion-Like Polymerization in Immunity and Inflammation
}

\author{
Xin Cai, ${ }^{1}$ Hui Xu, ${ }^{1}$ and Zhijian J. Chen ${ }^{1,2}$ \\ ${ }^{1}$ Department of Molecular Biology, University of Texas Southwestern Medical Center, Dallas, \\ Texas 75390-9148 \\ ${ }^{2}$ Howard Hughes Medical Institute, University of Texas Southwestern Medical Center, \\ Dallas, Texas 75390-9148 \\ Correspondence: xin.cai@utsouthwestern.edu; zhijian.chen@utsouthwestern.edu
}

\begin{abstract}
The innate immune system relies on receptors that sense common signs of infection to trigger a robust host-defense response. Receptors such as RIG-I and NLRP3 activate downstream adaptors mitochondrial antiviral signaling (MAVS) and apoptosis-associated speck-like protein (ASC), respectively, to propagate immune and inflammatory signaling. Recent studies have indicated that both MAVS and ASC form functional prion-like polymers to propagate immune signaling. Here, we summarize the biochemical, genetic, and structural studies that characterize the prion-like behavior of MAVS and ASC in their respective signaling pathways. We then discuss prion-like polymerization as an evolutionarily conserved mechanism of signal transduction in innate immunity in light of the similarity between the NLRP3-ASC, the NLRP3-ASC pathway in mammals, and the NWD2-HET-s pathway in fungi. We conclude by outlining the unique advantages to signaling through functional prions and potential future directions in the field.
\end{abstract}

\section{INNATE IMMUNE SIGNALING: SENSORS, ADAPTORS, AND EFFECTORS}

Thenters he innate immune system is an ancient and conserved defense mechanism against pathogen invasion. As the first line of host defense, it relies on numerous germline-encoded pattern recognition receptors to detect signs of infection or cellular danger to trigger a rapid, robust, and generic response for host protection (Takeuchi and Akira 2010). Conserved pathogenor danger-associated molecular patterns, such as bacterial lipopolysaccharide, foreign nucleic acids, and cytosolic DNA, activate innate im- mune sensors such as Toll-like receptors, retinoic acid-inducible gene I (RIG-I)-like receptors, nucleotide-binding oligomerization domain (NOD)-like receptors (NLRs), and the recently identified cyclic GMP-AMP synthase DNA sensor (Chen et al. 2009; Yoneyama and Fujita 2009; Casanova et al. 2011; Sun et al. 2013; Wu et al. 2013). Within minutes to hours of activation, the pattern recognition receptors trigger a myriad of signaling cascades that culminate in the production of protective chemokines and cytokines, which serve both as a rapid control of the insult and as a means of activating the more specific and longer-lasting adaptive

Editor: Stanley B. Prusiner

Additional Perspectives on Prion Biology available at www.cshperspectives.org

Copyright (C) 2017 Cold Spring Harbor Laboratory Press; all rights reserved; doi: 10.1101/cshperspect.a023580

Cite this article as Cold Spring Harb Perspect Biol 2017;9:a023580 
X. Cai et al.

immune response (Iwasaki and Medzhitov 2010).

Following ligand recognition, each pattern recognition receptor activates a downstream adaptor protein that forms an oligomeric signalosome for signal transduction. For instance, Toll-like receptors, RIG-I-like receptors, NLRs, and cyclic GMP-AMP synthase activate the respective downstream adaptors MyD88/TRIF, mitochondrial antiviral signaling (MAVS) (also known as VISA, IPS-1, or CARDIF), apoptosis-associated speck-like protein (ASC), and STING (also known as MITA, MPYS, or ERIS) to propagate immune and inflammatory responses. Each adaptor protein harbors distinct protein interaction domains that are essential for bridging the upstream receptors to their downstream effectors. In this regard, the death domain (DD) superfamily is one of the most common protein domains observed in innate immunity, inflammation, and cell death (Park et al. 2007). Members of the DD superfamily are defined by their shared structural features, including a unique fold composed of six $\alpha$-helices. The caspase activation and recruitment domain (CARD), PYRIN, death effector domain, and DD subfamilies make up the DD superfamily, and each subfamily mediates signal transduction through homotypic interactions and the formation of oligomeric complexes.

\section{The RIG-I Antiviral Pathway Signals through} CARD Polymerization

The RIG-I-like receptors include RIG-I, MDA5, and LGP2, which detect cytosolic viral RNA to activate the downstream adaptor protein MAVS (Fig. 1). As the best-characterized RIG-I-like receptor, RIG-I contains N-terminal tandem CARDs (RIG-I 2CARD), a middle helicase domain, and a C-terminal regulatory domain. Bioinformatics analysis indicated that the RIG-I helicase shares homology with the endoribonuclease Dicer, which plays an essential role in RNA interference, a primordial form of antiviral immunity (Zou et al. 2009). Accumulating evidence suggests that RIG-I-like helicases are evolutionarily conserved and serve antiviral functions from Caenorhabditis elegans to mammals (Deddouche et al. 2008; Ashe et al. 2013).

In mammals, RIG-I is activated by binding to viral RNAs bearing $5^{\prime}$ triphosphates $\left(5^{\prime}\right.$-ppp) or disphosphates $\left(5^{\prime}-\mathrm{pp}\right)$, which distinguish them from $5^{\prime}$-capped cellular RNA (Hornung et al. 2006; Goubau et al. 2014). Ligand binding to its C-terminal regulatory domain releases RIG-I from an autoinhibited state and frees its N-terminal tandem CARDs for binding to lysine-63 (K63)-linked polyubiquitin (Ub) chains (Zeng et al. 2010). K63-polyubiquitin chain binding is essential for RIG-I activation, as it converts RIG-I into a signaling competent tetramer (Zeng et al. 2010; Jiang et al. 2012; Peisley et al. 2014). The RIG-I:RNA:Ub complex then activates the downstream adaptor MAVS, which also harbors an N-terminal CARD (MAVS $^{\text {CARD }}$ ). Interactions between the CARDs of RIG-I and MAVS convert MAVS from an inactive, autoinhibited monomer into active prion-like filaments, the core of which is composed of polymerized MAVS CARD subunits (Hou et al. 2011; Cai et al. 2014a; Shi et al. 2015). Functional and active MAVS fibers then propagate downstream signaling by recruiting the kinases TBK1 and IKK, which in turn activate the transcription factors IRF3 and NF- $\kappa \mathrm{B}$, respectively, to induce the production of antiviral type-I interferons (Fig. 1) (Zeng et al. 2009; Liu et al. 2013, 2015).

The Inflammasomes: Model of PYRIN Domain Polymerization

The inflammasomes is another important DDcontaining signaling complex, which is formed when NLRs are activated by pathogens and other noxious stimuli (Martinon et al. 2002; Lamkanfi and Dixit 2014). Here, NLRs, such as NLRP3 and the DNA receptor AIM2, activate the adaptor protein ASC to propagate a proinflammatory response (Fig. 1). The exact trigger for NLRP3 activation remains unclear. Both AIM2 and NLRP3 contain N-terminal PYRIN domains that interact with the PYRIN domain of ASC (ASC ${ }^{\mathrm{PYD}}$ ), thereby converting ASC into functional prion-like filaments (Cai et al. 2014a; $\mathrm{Lu}$ et al. 2014). In addition to an $\mathrm{N}$-terminal 
Prion-Like Polymerization in Immunity and Inflammation

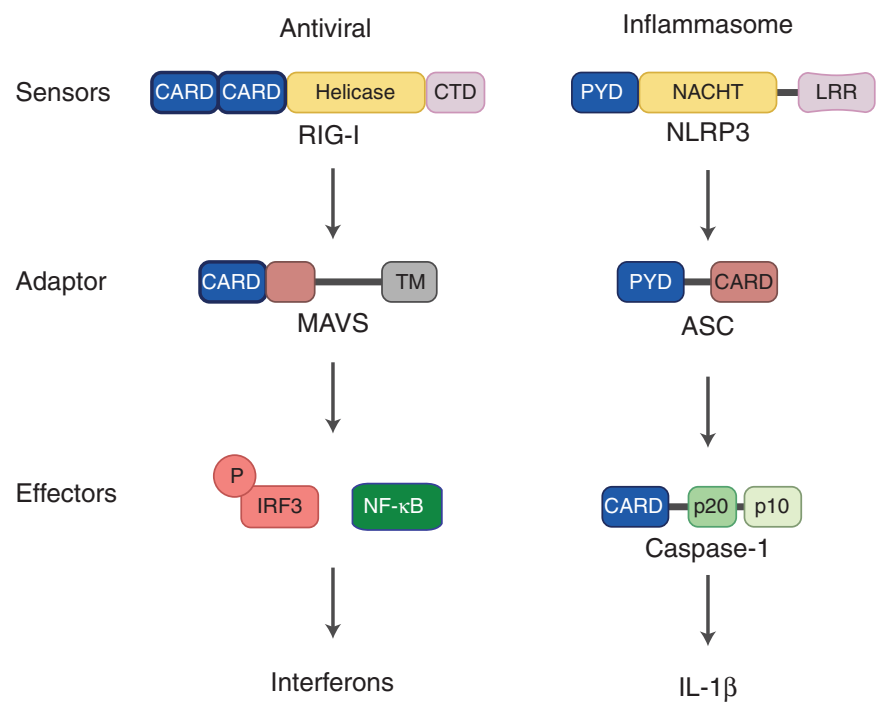

Figure 1. Cartoon depictions of the sensors, adaptors, and effectors in the mitochondrial antiviral signaling (MAVS)-dependent antiviral and apoptosis-associated speck-like protein (ASC)-dependent inflammasome pathways. Both MAVS and ASC serve as key adaptor proteins that relay multiple upstream signals to downstream effectors. The cytosolic RNA sensor RIG-I activates the MAVS protein to induce the production of interferon through the transcription factor IRF3 and NF-кB. Similarly, NLRP3 activates the adaptor ASC to produce proinflammatory cytokines such as IL-1 $\beta$. Caspase- 1 activation also leads to cell death (pyroptosis).

PYRIN that forms fibers, ASC also contains a Cterminal CARD that propagates downstream signaling by binding to the CARD of pro-caspase-1. Active ASC filaments then recruit multiple pro-caspase-1 proteins, which are brought into proximity to trigger their autoactivation. Mature caspase- 1 then converts pro-interleukin (IL) $-1 \beta$ into the mature IL- $1 \beta$ that is secreted by the cell.

\section{MAVS IS A BENEFICIAL MAMMALIAN PRION-LIKE PROTEIN}

Initial Identification and Characterization of MAVS

Early studies of RIG-I revealed that it contains tandem N-terminal CARDs, which are essential for RIG-I signaling (Yoneyama et al. 2004). MAVS also contains an N-terminal CARD that interacts with RIG-I 2CARD (Kawai et al. 2005; Meylan et al. 2005; Seth et al. 2005; Xu et al. 2005). Initial studies revealed that unlike other innate immune-signaling proteins or adaptors,
MAVS was unique in two regards. First, it was found to be tail-anchored to the outer membrane of the mitochondria through its C-terminal transmembrane domain (Seth et al. 2005). Second, activation of MAVS converted the protein into a detergent-insoluble form that is resistant to extraction by $0.5 \%$ Triton X-100 (Seth et al. 2005).

MAVS provides a direct link between immune signaling and mitochondria, an organelle of bacterial origin. For reasons that remain unclear, mitochondrial localization of MAVS is essential for its function, because deletion of its transmembrane domain completely abolished MAVS signaling. For instance, viruses such as hepatitis $\mathrm{C}$ have evolved proteases that specifically cleave MAVS off the mitochondrial membrane to prevent a host immune response (Li et al. 2005; Meylan et al. 2005). Mice deficient in MAVS were unable to produce type-I interferons (Sun et al. 2006). These examples highlight the essential role of MAVS and its mitochondrial localization in antiviral immunity. 
X. Cai et al.

\section{Biochemical Characterization of MAVS as a Prion}

To further characterize the active form of MAVS, subsequent biochemical studies found that following virus infection, endogenous MAVS formed very large particles (greater in size than the $26 \mathrm{~S}$ proteasome), as analyzed by sucrose density gradient ultracentrifugation (Hou et al. 2011). The large MAVS particles were found to be resistant to $2 \%$ sodium dodecyl sulfate (SDS), as revealed by high molecular weight smears on the semi-denaturing detergent agarose gel electrophoresis (SDD-AGE) assay, which is commonly used for analyses of prions (Alberti et al. 2010). Only the SDS-resistant MAVS polymers, but not soluble MAVS proteins, were capable of activating the downstream transcription factor IRF3 (Hou et al. 2011). Under the electron microscope (EM), purified active MAVS particles formed homogenous fibers with a striking resemblance to those formed by a fragment of the mammalian prion protein $(\mathrm{PrP})$. Proteinase-K digestion of MAVS fibers revealed a resistant fiber core composed of MAVS $^{\text {CARD }}$, indicating that MAVS ${ }^{\text {CARD }}$ is the prion domain that mediates its assembly into filaments (Hou et al. 2011).

One of the defining features of prions is their ability to form self-perpetuating structural conformations that convert the native form of the protein into the prion form. Indeed, brief incubation of a substoichiometric amount of MAVS ${ }^{\text {CARD }}$ fibers converts endogenous MAVS into large, SDS-resistant polymers that are capable of activating the IRF3 transcription factor, much like the biochemical switch observed in MAVS following viral infection (Hou et al. 2011). High-resolution imaging of cells indicated that endogenous MAVS formed fibrous polymers near the mitochondria following viral infection (Xu et al. 2014). Together, these results indicated that MAVS switches into self-perpetuating prion-like fibers to propagate innate immune signaling.

\section{Genetic Validation of MAVS as a Prion}

Subsequent genetic studies in yeast further supported MAVS as a bona fide, gain-of-function prion. Yeast contains more than a dozen prionlike proteins and is arguably the best model organism to study prions given the abundance of well-established prion assays and genetic tools (Alberti et al. 2010). One of the bestcharacterized yeast prions is the SUP35 protein, which contains an $\mathrm{N}$-terminal prion domain $\left(\mathrm{NM}\right.$ or SUP $35^{\mathrm{NM}}$ ) and a C-terminal catalytic domain that carries out its translation termination function (Sup35C) (Tuite and Serio 2010). Using the SUP35 yeast prion assay, in which the prion domain of SUP35 (SUP35 ${ }^{\mathrm{NM}}$ ) was replaced with MAVS ${ }^{\mathrm{CARD}}$, we showed that MAVS $^{\text {CARD }}$ faithfully recapitulated the key prion properties of SUP3 $35^{\mathrm{NM}}$ in yeast (Cai et al. 2014a). Specifically, transient expression of MAVS ${ }^{\text {CARD }}$ converts the MAVS ${ }^{\text {CARD }}$-SUP35C fusion protein into a prion that is dominant, cytoplasmically inherited, and transmissible through numerous cell divisions. Mutagenesis experiments suggest that MAVS prion conversion occurs through separable nucleation and polymerization steps, much like other prions identified to date.

\section{RIG-I DIRECTLY NUCLEATES MAVS PRION CONVERSION}

Unlike other prion proteins that are stochastically switched into the polymer forms, MAVS undergoes signal-dependent prion conversion regulated by its heterotypic upstream signaling protein, RIG-I. Central to our understanding of MAVS activation is the mechanistic dissection of how RIG-I nucleates MAVS prion conversion. Although the activation of full-length RIG-I requires the sequential binding of both RNA (to the C-terminal regulatory domain) and K63 poly-Ub (to RIG-I 2CARD), truncation studies indicated that RIG-I 2CARD in complex with poly-Ub is sufficient to activate MAVS in vitro and in cells (Zeng et al. 2010). Consistently, within minutes of incubation with crude mitochondria containing endogenous MAVS, a substoichiometric amount of active RIG-I 2CARD:polyUb complex induces nearly complete conversion of MAVS into functional polymers that are capable of downstream signaling (Hou et al. 2011). 
In yeast that is not known to express a RIGI-like pathway, transient expression of RIG-I 2CARD robustly induces MAVS ${ }^{\text {CARD }}$-SUP35C polymerization, indicating that RIG-I 2CARD is sufficient to directly nucleate MAVS prion conversion (Cai et al. 2014a). The frequency of the MAVS ${ }^{\text {CARD }}$ prion switch induced by RIG-I 2CARD expression was several orders of magnitude greater than that induced by MAVS CARD expression, suggesting that RIG-I 2CARD is a much more potent nucleation factor. Point mutations in MAVS ${ }^{\text {CARD }}$ that disrupted RIG-I 2CARD-induced prion formation in yeast also abolished its signaling activity in mammalian cells following viral infection. Together, these biochemical and genetic studies establish MAVS as a bona fide gain-of-function mammalian prion essential for the propagation of RIG-I antiviral signaling.

\section{STRUCTURAL STUDIES OF MAVS FILAMENT REVEALED A SINGLE-TURN HELICAL FIBER NUCLEATED BY THE RIG-I 2CARD:K63-UB2 COMPLEX}

Recent structural studies of MAVS ${ }^{\mathrm{CARD}}$ filaments and active RIG-I 2CARD in complex with K63 ubiquitin chains have begun to shed light on the mechanisms of MAVS activation and polymerization. The crystal structure of inactive MAVS ${ }^{\text {CARD }}$ monomer is consistent with the characteristic six $\alpha$-helical bundle fold that is shared by members of the DD superfamily (Potter et al. 2008). Two recent cryo-electron microscopy (cryo-EM) structural models of MAVS ${ }^{\text {CARD }}$ fibers using proteins purified from bacteria and mammalian cells have emerged. The two studies at 3.6- and 4.2- $\AA$ resolution revealed left-handed helical filaments with $\mathrm{C} 1$ symmetry composed of individual MAVS CARD subunits stacked on top of one another ( $\mathrm{Wu}$ et al. 2014; Xu et al. 2014, 2015). Interestingly, the transition from the soluble to the prion form of MAVS did not involve any $\alpha$-helical to $\beta$-sheet structural changes, as typically observed in other prion conversions. In addition, both cryo-EM models did not reveal any major structural alterations of MAVS ${ }^{\text {CARD }}$ in its monomeric or polymerized form. Consistently, MAVS fibers failed to stain with thioflavin $\mathrm{T}$ (ThT), which typically binds to $\beta$-amyloids.

Although the structure of the full-length RIG-I:RNA:Ub complex remains to be determined, recent effort has revealed the structure of the RIG-I 2CARD and K63 di-ubiquitin complex, the minimal subunit that is necessary and sufficient for MAVS activation. X-ray crystallography of the RIG-I 2CARD:Ub2 complex revealed a single-turn helical tetramer formed by RIG-I 2CARD, stabilized by interactions with K63 di-ubiquitin at the periphery (Peisley et al. 2014). The active RIG-I 2CARD:Ub2 complex resembles a lock-washer that is displaced by half its thickness or one CARD subunit (RIG-I contains two CARDs) at the end of the complete turn, which is ideally positioned to nucleate MAVS ${ }^{\mathrm{CARD}}$ polymerization. To characterize RIG-I 2CARD-mediated MAVS CARD nucleation, X-ray crystallography studies of a RIG-I 2CARD-MAVS ${ }^{\text {CARD }}$ fusion protein indicated that the MAVS ${ }^{\mathrm{CARD}}$ monomers polymerized following the trajectory set by the RIG-I 2CARD:Ub nucleus, which is consistent with the structures of active MAVS ${ }^{\text {CARD }}$ filament made up of a single-stranded helical filament (Wu et al. 2014).

\section{Model of RIG-I-MAVS Signaling}

The following proposed model of RIG-I-MAVS signaling summarizes the key findings described above (Fig. 2). (1) Ligand binding: RNA virus infection of cells generates $5^{\prime}$-ppp RNA ligands that bind to the C-terminal regulatory domain of RIG-I, bringing multiple RIG-I proteins into proximity and releasing RIG-I N-terminal CARDs from an autoinhibited state. (2) MAVS nucleation: The binding of K63 polyubiquitin chains to the freed RIG-I 2CARD stabilizes the RIG-I:RNA complex into a signaling-competent tetrameric complex that directly nucleates MAVS prion conversion through CARD-CARD interactions. (3) MAVS polymerization: Following MAVS nucleation, irreversible and spontaneous polymerization ensues such that an all-or-none response is generated as long as the RIG-I stimulation threshold is reached. (4) Downstream signal transduc- 
X. Cai et al.
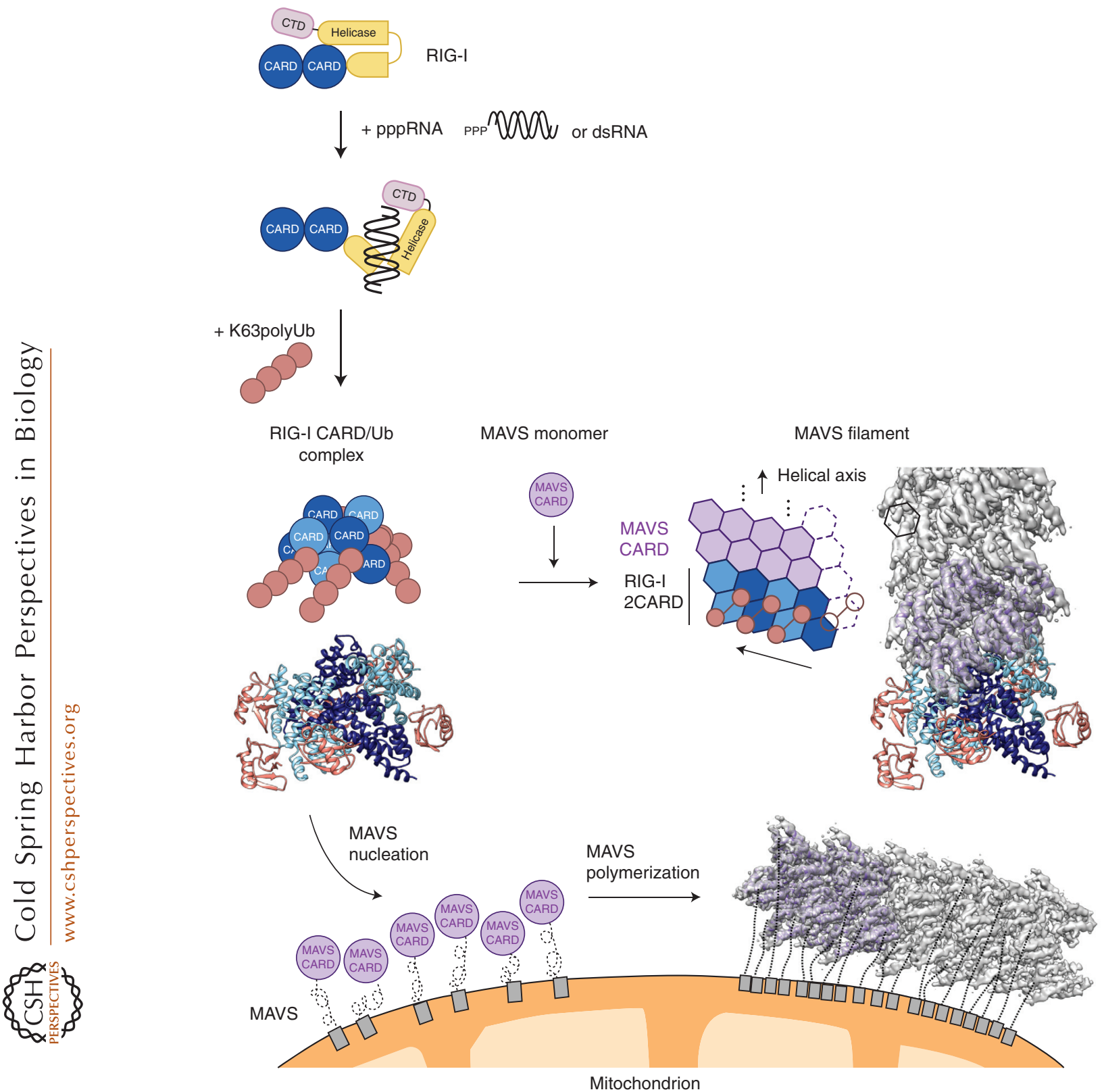

Figure 2. A proposed model for mitochondrial antiviral signaling (MAVS) activation by RIG-I-induced polymerization. Detection of viral 5'-ppp RNA or double-stranded (ds) RNA by RIG-I triggers a conformational change that exposes the N-terminal tandem caspase activation and recruitment domains (CARDs) of RIG-I (RIG-I 2CARD). RIG-I 2CARD then binds to K63-linked polyubiquitin chains, which facilitate the formation of a RIG-I tetramer. The RIG-I 2CARD tetramer interacts with MAVS CARD, providing a template to initiate the nucleation of a MAVS CARD filament. The MAVS CARD filament recruits additional MAVS CARD to form long filaments on the surface of the mitochondrial outer membrane. The MAVS filaments are competent in activating downstream signaling cascades to induce type-I interferons and other antiviral effectors. The models of RIG-I CARD/Ub/MAVS CARD complex and MAVS filaments are built based on PDB 4NQK, 4P4H, 2VGQ, and EMD-6428. 
tion: MAVS fibers are then able to recruit downstream signaling proteins to propagate a rapid and robust immune response.

\section{ASC MEDIATES INFLAMMATORY SIGNALING THROUGH PRION CONVERSION}

\section{Identification of ASC as a Functional Prion}

In an effort to identify other DD-containing signaling proteins that may have prion-like properties, a candidate screen on an additional 18 DDs was performed using the yeast SUP35based prion assay (Cai et al. 2014a). Each DD candidate was tested for its ability to functionally replace $\operatorname{Sup} 35^{\mathrm{NM}}$ and was expressed as a DD-SUP35C fusion in place of endogenous SUP35 in an ade1-14 genetic background. Yeast strains expressing ASC ${ }^{\mathrm{PYD}}$-SUP35C exhibited a high frequency of spontaneous prion conversion, as revealed by the formation of white colonies on complete (yeast extract peptone dextrose [YPD]) media. Transient expression of $\mathrm{ASC}^{\mathrm{PYD}}$ greatly enhanced the frequency of ASC $^{\mathrm{PYD}}$ prion conversion in yeast, consistent with properties of other prions (Cai et al. 2014a). Cytoduction and SDD-AGE analyses in yeast confirmed that the ASC ${ }^{\mathrm{PYD}}$ prion particles were cytoplasmically inherited and formed SDS-resistant polymers.

Like MAVS, ASC is activated by heterotypic upstream proteins such as NLRP3 and AIM2. Indeed, transient expression of AIM2 or NLRP $3^{\text {PYD }}$ induced robust ASC ${ }^{\text {PYD }}-S U P 35 \mathrm{C}$ prion conversion in yeast at a frequency several hundred folds greater than that observed with expression of ASC ${ }^{\text {PYD }}$ (Cai et al. 2014a). This again indicates that the physiologic upstream signaling receptors more potently nucleate ASC polymerization than overexpression of ASC $^{\text {PYD }}$. Point mutations that inhibited ASC prion conversion in yeast also abolished its activation by NLRP3 or AIM2 in mammalian cells, indicating that its prion conversion is essential for its signaling ability.

Under the electron microscope, recombinant $\mathrm{ASC}^{\mathrm{PYD}}$ protein formed filaments resembling those of MAVS ${ }^{\text {CARD }}$ (Cai et al. 2014a).
When incubated with cell lysates containing soluble, inactive ASC-green fluorescent protein (GFP), a substoichiometric amount of ASC ${ }^{\text {PYD }}$ fibers converted the ASC-GFP protein into large polymers that sedimented to the bottom of a sucrose density gradient after ultracentrifugation, indicating that $\mathrm{ASC} \mathrm{PYD}^{\mathrm{PY}}$ fibers were able to convert soluble ASC into self-perpetuating polymers (Cai et al. 2014a). Only the high molecular weight form of ASC-GFP was able to recruit and activate downstream pro-caspase-1. An ASC polymerization mutant remained in the low molecular weight fractions on sucrose gradient and was unable to recruit pro-caspase-1.

\section{Structural Studies of ASC Filaments}

The high-resolution cryo-EM structure of the ASC $^{\text {PYD }}$ fiber at $3.8 \AA$ revealed a three-start, right-handed helical filament (Lu et al. 2014). Analogous to MAVS ${ }^{\text {CARD }}$ filaments, no $\alpha$-helical to $\beta$-sheet transition was observed between the monomeric and polymerized form of $\mathrm{ASC}^{\mathrm{PYD}}$, and each subunit remained largely unchanged during the prion switch. In vitro ASC $^{\text {PYD }}$ polymerization assay using goldlabeled $\mathrm{AIM} 2^{\mathrm{PYD}}$ or $\mathrm{NLRP} 3^{\mathrm{PYD}-\mathrm{NBD}}$ showed that each receptor localized to the end of the ASC $^{\text {PYD }}$ filament, consistent with their role as nucleation factors (Lu et al. 2014). At times, several gold-bound AIM2 ${ }^{\mathrm{PYD}}$ proteins co-localized to one end of the filament, consistent with subsequent studies, and the model that limited oligomerization of upstream receptors precedes the nucleation and polymerization of ASC (Morrone et al. 2015).

\section{ASC Prion Particles Have "Infectious" Properties}

As further support for the prion model of ASC activation, cell-based and mouse studies indicate that activated ASC particles are released from dying cells and taken up by neighboring macrophages, leading to nucleation of soluble, endogenous ASC proteins to perpetuate inflammasome signaling (Baroja-Mazo et al. 2014; Franklin et al. 2014). Intraperitoneal injection 
X. Cai et al.

of active ASC particles resulted in a proinflammatory response only in mice that expressed endogenous ASC but not in ASC deficient mice, suggesting that ASC particles possess infectious properties that catalytically convert endogenous ASC into the prion form, much like mammalian PrP. In mice, ASC particles were also found to be stable in the extracellular space for 96 hours, indicating that they are relatively resistant to protease inactivation, much like other prions.

\section{UNIQUE PROPERTIES OF PRIONS FUNCTIONING IN INNATE IMMUNITY}

Although MAVS and ASC share the key biochemical and genetic features of prions with other known prion-like proteins, they differ in several important aspects. First, unlike most prions that confer loss of function, MAVS and ASC are both gain-of-function prions. Their prion conversion is central to their function. For instance, cells containing MAVS mutants defective in prion formation were phenotypically identical to mavs $^{-1-}$ cells in response to viral infection (Liu et al. 2013). Consistently, MAVS and ASC polymerization mutants were unable to recruit essential downstream signaling proteins. Second, the monomeric forms of MAVS ${ }^{\text {CARD }}$ and ASC ${ }^{\text {PYD }}$ are well-folded globular domains that are not enriched in glutamine and asparagine amino acids, which are often found in prion domains. X-ray crystallography and NMR studies revealed that both proteins form $\alpha$-helical bundles in their native states, unlike the unstructured prion domains often found in other aggregation-prone proteins (Liepinsh et al. 2003; Potter et al. 2008). Third, MAVS and ASC prion conversion lacked $\beta$-amyloid transitions usually observed in other prions. Both prion domains maintained their $\alpha$-helical folds in their respective fibers, which are formed by the polymerization of individual $\alpha$-helical subunits. Consistently, neither filament stained with ThT, as would be expected for a $\beta$-amyloid. Fourth, unlike most prions that are formed stochastically, MAVS and ASC undergo regulated prion conversion, in which activation of their corresponding upstream sig- naling partner initiates their polymerization. In other words, their prion conversion is regulated and can be quickly switched on by the appropriate stimulus.

\section{A PARALLEL NLRP3-ASC INFLAMMASOME- LIKE PATHWAY IN FUNGI}

MAVS and ASC Share Properties with the Fungal Prion HET-s

As gain-of-function prions, MAVS and ASC are similar in this regard to the fungal prion HET-s (Fig. 3). Similar to HET-s, MAVS and ASC do not stain with ThT or require Hsp104 for propagation of their prions. HET-s triggers cell death by converting its allelic variant HET-S (big "S") into a pore forming toxin (Saupe 2011; Seuring et al. 2012). HET-s and HET-S are both 289amino-acid proteins that differ at only 13 residues (for review, see Riek and Saupe 2016). Both proteins contain N-terminal HeLo domains and interchangeable $\mathrm{C}$-terminal prion domains $(\mathrm{PrD})$ that polymerize into a $\beta$-solenoid. HET-s mediated HET-S activation has been proposed as a form of immune response that prevents the horizontal transfer of infectious pathogens during fungal cell fusion. Specifically, cell death occurs upon cell fusion only when one cell contains the prion form of HET-s, which then nucleates the structural conversion of soluble HET-S expressed by the other cell. Following polymerization of its $\mathrm{PrD}$, the HeLo domain of HET-S forms a membrane-permeable toxin to induce death of the fused cell (Mathur et al. 2012; Seuring et al. 2012). Mutations in the HET-s HeLo domain, however, have rendered the protein inactive, leaving its $\operatorname{PrD}$ as the only functional domain. Although the two proteins share a near identical prion domain (with only a 3-amino-acid difference), the mechanism of HET-s prion conversion had been largely unclear until recent studies.

NWD2 and HET-s Are Fungal Homologs of Mammalian NLRP3 and ASC

A recent bioinformatic search for fungal proteins that share a similar amino acid sequence 
Mammals

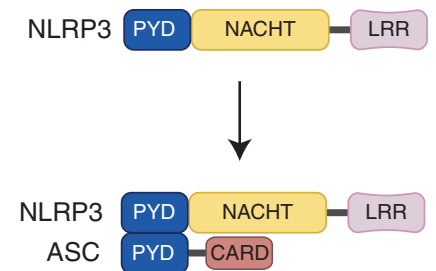

ASC

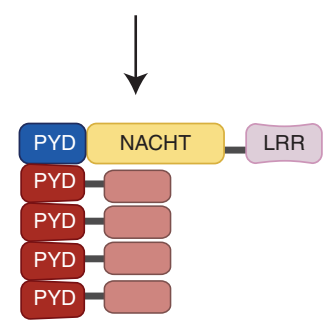

Fungi
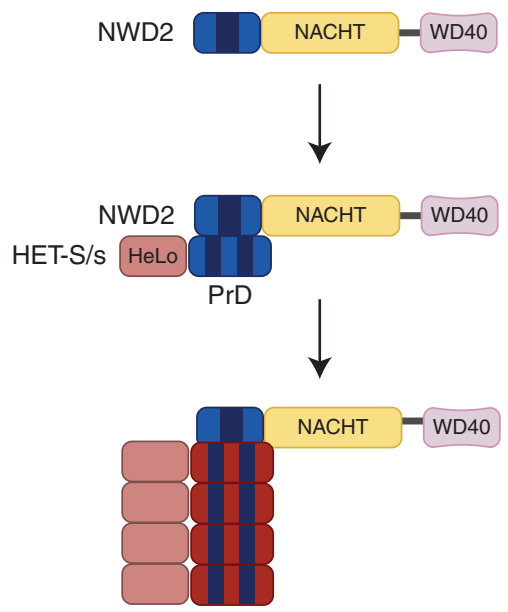

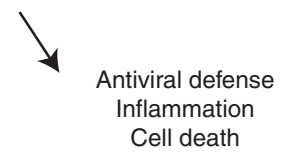

Figure 3. Homologous mammalian and fungal host-defense pathways that signal through prion conversion. In mammals, receptors such as NLRP3 sense pathogen invasion or cell damage to rapidly convert its downstream apoptosis-associated speck-like protein (ASC) into its active prion form, which then leads to cytokine secretion and cell death to protect the host. A homologous pathway is present in filamentous fungi, where activated NWD2 receptor converts the HET-S/s protein into a prion to induce cell death. The fungal NWD2-HET-S/s pathway is remarkably similar to the mammalian NLRP3-ASC pathway in both function and domain organization, suggesting that signaling through self-perpetuating protein conformations for host defense and cell-fate determination is conserved from fungi to mammals.

as the HET-s PrD identified NWD2, a protein that strikingly resembles mammalian NLRP3 in domain organization and is encoded by a gene immediately adjacent to het-S (Fig. 3) (Daskalov et al. 2012). Notably, the NWD2 N-terminus contains an approximate 30-amino-acid fragment $\left(\mathrm{NWD} 2^{\mathrm{N} 30}\right)$ that is predicted to take on a similar structural fold as the HET-s prion domain, much like the structural relationship between NLRP3 ${ }^{\mathrm{PYD}}$ and $\mathrm{ASC}^{\mathrm{PYD}}$. In addition, both NWD2 and NLRP3 contain middle NACHT domains that mediate protein oligomerization. In its C-terminus, NWD2's WD40 repeat may serve as a ligand-binding domain, similar to the proposed function of NLRP3's leucine-rich repeat.

Immunofluorescence studies in mammalian cells indicated that transient expression of the $\mathrm{NWD}^{\mathrm{N} 30}-\mathrm{NLRP}^{\triangle \mathrm{PYD}}$ fusion protein (where NLRP3 ${ }^{\text {PYD }}$ is replaced by $\mathrm{NWD} 2^{\mathrm{N} 30}$ ), but neither NLRP3 wild-type (WT) nor $\mathrm{NWD}^{\mathrm{N} 30}$ alone converted HET-s ${ }^{\mathrm{PrD}}$ from a diffuse cytoplasmic pattern to a large, distinct focus (Cai et al. 2014a). This result indicated that $\mathrm{NWD} 2{ }^{\mathrm{N} 30}$ can indeed nucleate the polymerization of HET-s ${ }^{\mathrm{PrD}}$. However, the interaction between NWD2 and HET-s depended on the oligomerization of NWD2, which occurred when $\mathrm{NWD}_{2}{ }^{\mathrm{N} 30}$ was fused to $\mathrm{NLRP}^{\triangle \mathrm{PYD}}$ (which contains the NACHT domain) but not when expressed alone. Functionally, we found that fungal $\mathrm{NWD}^{\mathrm{N} 30}$ and HET-s ${ }^{\mathrm{PrD}}$ can replace the PYRINs of NLRP3 and ASC, respectively, in mammalian inflammasome signaling (Cai et al. 2014a). Specifically, in response to nigericin, a chemical that activates the NLRP3 pathway, only $\mathrm{NWD}^{\mathrm{N} 30}-\mathrm{NLRP}^{\Delta \mathrm{PYD}}$ fusion, but not WT NLRP3, was able to nucleate the poly- 
X. Cai et al.

merization of HET-s ${ }^{\mathrm{PrD}}-\mathrm{ASC}^{\Delta \mathrm{PYD}}$ (where ASC $^{\text {PYD }}$ was replaced by HET-s ${ }^{\text {PrD }}$ ) to activate caspase- 1 . In contrast, WT ASC polymerization was induced by activation of NLRP3, but not the $\mathrm{NWD}^{\mathrm{N} 30}-\mathrm{NLRP} 3^{\mathrm{APD}}$ fusion protein. These results indicate that the structure-activity relationship between NWD2 ${ }^{\mathrm{N} 30}$ and HET$s^{\mathrm{PrD}}$ is interchangeable with that between NLRP $3^{\text {PYD }}$ and ASC ${ }^{\text {PYD }}$, where each distinct prion domain can be nucleated only by the effector domain of its cognate upstream receptor.

Subsequent studies in the fungus Podospora anserina revealed that oligomerized NWD2 induced HET-s prion conversion (Daskalov et al. 2015). Specifically, in fungi expressing chimeric NWD2 variants harboring foreign WD40 repeats, HET-s prion formation was induced by exposure to the respective ligand for each fused WD40 repeat. $\mathrm{NWD}^{\mathrm{N} 30}$ was found to be responsible for its HET-s templating activity, because mutations predicted to affect its $\beta$ solenoid fold abolished its ability to nucleate HET-s polymerization. In fungi, expression of NWD $2^{\mathrm{N} 30}-\mathrm{RFP}$ induced the relocalization of HET-S to the cell periphery and activated the pore-forming protein, providing evidence of signaling interaction between NWD2 and HET-s/S in their original cellular context.

The homology between the NWD2-HET-s and NLRP3-ASC pathways suggests that signaling through prion-like polymerization is evolutionarily conserved, especially in pathways responsible for self-defense or fate switch (Fig. 3). Specifically, both pathways rely on distinct domains in adaptor proteins that undergo transmissible structural changes nucleated by upstream receptors, leading to signal amplification that eventually results in cell death. In addition to the similarities between NLRP3 and NWD2, there are several other reasons to suggest that the NLRP3-ASC-pro-caspase-1 and other inflammasome pathways may have evolved from the NWD2-HET-s/HET-S pathway. With a nonfunctional HeLo domain, HET-s essentially serves as a self-perpetuating prion adaptor for activation of HET-S through interactions between their PrDs. Following activation by HET-s, HET-S is then able to execute cell death through its functional HeLo domain.
In mammals, ASC has evolved into a bipartite PYRIN-CARD adaptor and serves similar functions as HET-s to bridge NLRP3 and procaspase- 1 activation. Like HET-S, pro-caspase1 harbors an $\mathrm{N}$-terminal CARD for interaction with ASC and a C-terminal executioner domain that contains all of its catalytic function. In the absence of HET-s, NWD2 might directly activate HET-S, which shares interchangeable PrDs with HET-s. Likewise, in mammalian inflammasome signaling, a number of NLRs, such as NLRC4, can bypass ASC and directly activate pro-caspase-1. Recent cryo-EM studies indicated that NLRC4 is also activated through a selfcatalytic conformational switch that results in the formation of a helical wheel with its upstream activator, NLR apoptosis inhibitory protein (NAIP) (Hu et al. 2015). The NLRC4 polymer can then either activate ASC or directly recruit pro-caspase-1.

As further evidence for the evolutionary conservation of the prion-like polymerization as a signaling mechanism in cell death pathways, a recent study found that mammalian necrosis mediated by RIP1/RIP3 (upstream kinase) and MLKL (cell death effector) also shares features with an NWD2/HET-S-like pathway in fungi (Daskalov et al. 2016). To characterize other NWD2 and HET-S analogs, Daskalov et al. identified another gene cluster that contains an NLR and an adjacent effector harboring a prion domain — namely, PNT1 and HELLP, respectively - in addition to a putative lipase, SBP. PNT1, HELLP, and SBP each contains a PP motif, which is capable of amyloid conversion and shares sequence features with the RIP homotypic interaction motif (RHIM) domains of RIP1/RIP3 that had previously been observed to form amyloid fibrils ( $\mathrm{Li}$ et al. 2012). As a HET-S homolog, HELLP is activated by PPmotif-mediated polymerization and contains a HeLo-like domain to execute cell death. Remarkably, the HeLo-like domain of HELLP was found to be homologous with the MLKL $4 \mathrm{HB}$ domain, which is responsible for membrane permeation in mammalian necrosis (Sun et al. 2012; Cai et al. 2014b; Chen et al. 2014; Su et al. 2014). Consistently, prion conversion targets HELLP to the plasma membrane 
Prion-Like Polymerization in Immunity and Inflammation

to induce cell death. Taken together, these results suggest that mammalian necrosis and fungal cell death share homologous mechanisms mediated by evolutionarily conserved protein domains, where self-perpetuating prion-like polymerization governs programmed cell death.

\section{BENEFITS OF SIGNALING THROUGH PRION-LIKE POLYMERIZATION}

The finding that prion-like proteins mediate a protective innate immune response may be surprising at first. Until recently, the formation of prion-like aggregates in mammals had invariably been associated with pathologic processes, in which the host's homeostatic protective response had failed (Prusiner 1982; Jucker and Walker 2013; Ramaswami et al. 2013). Although the formation of large protein aggregates may be detrimental to individual cells, in the setting of a noxious insult that threatens the fitness of an organism, the formation of irreversible prions offers the host numerous advantages, as discussed in the following sections.

\section{Sensitive and Robust Signal Transduction Mechanism}

Signal transduction plays an essential role in all aspects of an organism's function. Although certain signals are beneficial, others serve as warnings of imminent danger. As the first line of defense against pathogen invasion, innate immune signaling plays an essential role in the well-being of the host. In this regard, our cells must evolve mechanisms to detect minute signs of pathogen invasion. Signal propagation through self-perpetuating prions offers the host a sensitive and robust response to minimal stimuli, in which the activation of a small number of proteins leads to a self-catalytic, all-ornone response. For instance, recent estimates suggest that the RIG-I/MAVS pathway can be activated by $\sim 20$ viral RNA molecules, much fewer than the number of RIG-I-like receptors in the cell (Zeng et al. 2010). In this case, as long as a sufficient number of RIG-I-like receptors can form an oligomer to nucleate MAVS, a full- scale response will follow, which ensures a complete response in the early phase of viral infection. In addition, the activation of MAVS not only leads to the production of type-I interferons, but also further amplifies the host response by inducing the expression of other self-defense genes such as RIG-I or NLRP3 and pro-IL-1 $\beta$, which are usually expressed at low levels in unstimulated cells.

\section{Altruistic Prions}

Viral and bacterial infections frequently lead to cell death, which prevents further pathogen replication and host damage. For instance, activation of the NLRP3 inflammasome triggers a poorly understood form of cell death, known as pyroptosis. The activation of cell death in conjunction with prion-like polymerization is unlikely to be a coincidence. These two mechanisms likely evolved to accommodate each other, allowing infected cells to secrete host-protective signals before undergoing senescence. Unlike in unicellular yeast, where prion formation has been proposed as a mechanism of phenotypic diversification to increase the fitness of each cell, altruistic prions such as MAVS and ASC enhance overall host fitness at the expense of individual cells. Interestingly, loss-of-function phenotypes govern most if not all yeast prions, whereas gain-of-function and beneficial prions have only been observed in multicellular organisms, such as filamentous fungi and mammals.

\section{Formation of Signalosomes}

Formation of large polymers offers spatial and temporal control over signal transduction. Activated MAVS and ASC form large signalosomes that can be visualized as distinct puncta or rods in cells (Broz et al. 2010; Xu et al. 2014). The formation of these large signalosomes is essential to recruit and activate downstream signaling proteins. For example, mutations that abolish MAVS or ASC polymerization also prevent the recruitment of downstream tumor necrosis factor (TNF) receptor-associated factors (TRAFs) or pro-caspase-1, respectively. Here, the forma- 
X. Cai et al.

tion of large polymers increases the local concentration of signaling molecules, thereby enhancing an otherwise low affinity interaction between MAVS and TRAFs or ASC and procaspase-1. By forming large subcellular structures, MAVS and ASC fibers serve as hubs to recruit downstream proteins to the appropriate location for their activation.

\section{CONCLUDING REMARKS AND FUTURE DIRECTION}

Studies of innate immune signaling uncovered a surprisingly conserved mechanism of signal transduction that utilizes prion-like switches to relay signals from divergent upstream sensors to downstream effectors. Although these early studies have mostly focused on the activation of MAVS and ASC, many important questions remain. For instance, the mitochondrial localization of MAVS is essential for its prion-like activity. When expressed as a cytosolic protein, MAVS $^{\text {CARD }}$ is unable to form prion-like oligomers, suggesting that MAVS polymerization is either promoted by the mitochondria or inhibited by the cytosol through unknown mechanisms. Future studies on the contribution of mitochondria to the prion-like activity of MAVS are essential for understanding the link between mitochondria and immunity. In addition, MAVS and ASC fibers must undergo cycles of seeding, polymerization, and fragmentation to stably propagate through numerous cell divisions. Essential factors and chaperones for MAVS and ASC propagation remain to be identified, because both proteins do not depend on Hsp104, which normally governs the fragmentation of most yeast prions. Understanding the proteins that promote MAVS and ASC prion propagation may also yield pathogen virulence targets against the immune system. Last, recent studies suggest the existence of mechanisms to prevent continuous signaling from MAVS prions. Specifically, we found that cell extracts from virus-infected cells were refractory to activation by active MAVS prion particles, suggesting the existence of downstream mechanisms to prevent continuous signaling from MAVS prion particles (Hou et al. 2011). Like- wise, mice infected with RNA virus do not produce interferons indefinitely. Indeed, the phenomenon of refractory immune tolerance has been described. For instance, prior exposure to endotoxin renders cells refractory to subsequent endotoxin stimulation, pointing to potential mechanisms that turn off innate immune signaling downstream from the adaptor proteins (Biswas and Lopez-Collazo 2009). Because unregulated MAVS or ASC signaling would result in autoimmune or autoinflammatory diseases, mechanisms must exist that appropriately shut off the signaling from these protein fibers. Although MAVS and ASC represent the first examples of functional mammalian prions, they are unlikely to be the only ones. As prion-like polymerization offers the host many unique advantages, this mechanism is also likely to be used by other biological pathways.

\section{ACKNOWLEDGMENTS}

Research in our laboratory is supported by grants from the National Institutes of Health (AI-93967), the Welch Foundation (I-1389), and the Cancer Research Prevention Institute of Texas (RP120718 and RP120718). Z.J.C. is a Howard Hughes Medical Institute (HHMI) Investigator.

\section{REFERENCES}

${ }^{*}$ Reference is also in this collection.

Alberti S, Halfmann R, Lindquist S. 2010. Biochemical, cell biological, and genetic assays to analyze amyloid and prion aggregation in yeast. Methods Enzymol 470: 709734.

Ashe A, Bélicard T, Le Pen J, Sarkies P, Frézal L, Lehrbach NJ, Félix MA, Miska EA. 2013. A deletion polymorphism in the Caenorhabditis elegans RIG-I homolog disables viral RNA dicing and antiviral immunity. eLife 2: e00994.

Baroja-Mazo A, Martín-Sánchez F, Gomez AI, Martínez CM, Amores-Iniesta J, Compan V, Barberà-Cremades M, Yagüe J, Ruiz-Ortiz E, Antón J, et al. 2014. The NLRP3 inflammasome is released as a particulate danger signal that amplifies the inflammatory response. Nat Immunol 15: 738-748.

Biswas SK, Lopez-Collazo E. 2009. Endotoxin tolerance: New mechanisms, molecules and clinical significance. Trends Immunol 30: 475-487.

Broz P, Newton K, Lamkanfi M, Mariathasan S, Dixit VM, Monack DM. 2010. Redundant roles for inflammasome 
receptors NLRP3 and NLRC4 in host defense against Salmonella. J Exp Med 207: 1745-1755.

Cai X, Chen J, Xu H, Liu S, Jiang QX, Halfmann R, Chen ZJ. 2014a. Prion-like polymerization underlies signal transduction in antiviral immune defense and inflammasome activation. Cell 156: 1207-1222.

Cai Z, Jitkaew S, Zhao J, Chiang HC, Choksi S, Liu J, Ward Y, Wu LG, Liu ZG. 2014b. Plasma membrane translocation of trimerized MLKL protein is required for TNF-induced necroptosis. Nat Cell Biol 16: 55-65.

Casanova JL, Abel L, Quintana-Murci L. 2011. Human TLRs and IL-1Rs in host defense: Natural insights from evolutionary, epidemiological, and clinical genetics. Annu Rev Immunol 29: 447-491.

Chen G, Shaw MH, Kim YG, Nuñez G. 2009. NOD-like receptors: Role in innate immunity and inflammatory disease. Annu Rev Pathol 4: 365-398.

Chen X, Li W, Ren J, Huang D, He WT, Song Y, Yang C, Li W, Zheng X, Chen P, et al. 2014. Translocation of mixed lineage kinase domain-like protein to plasma membrane leads to necrotic cell death. Cell Res 24: 105-121.

Daskalov A, Paoletti M, Ness F, Saupe SJ. 2012. Genomic clustering and homology between HET-S and the NWD2 STAND protein in various fungal genomes. PloS ONE 7: e34854.

Daskalov A, Habenstein B, Martinez D, Debets AJ, Sabaté R, Loquet A, Saupe SJ. 2015. Signal transduction by a fungal NOD-like receptor based on propagation of a prion amyloid fold. PLoS Biol 13: el002059.

Daskalov A, Habenstein B, Sabaté R, Berbon M, Martinez D, Chaignepain S, Coulary-Salin B, Hofmann K, Loquet A, Saupe SJ. 2016. Identification of a novel cell death-inducing domain reveals that fungal amyloid-controlled programmed cell death is related to necroptosis. Proc Natl Acad Sci 113: 2720-2725.

Deddouche S, Matt N, Budd A, Mueller S, Kemp C, GalianaArnoux D, Dostert C, Antoniewski C, Hoffmann JA, Imler JL. 2008. The DExD/H-box helicase Dicer-2 mediates the induction of antiviral activity in drosophila. Nat Immunol 9: 1425-1432.

Franklin BS, Bossaller L, De Nardo D, Ratter JM, Stutz A, Engels G, Brenker C, Nordhoff M, Mirandola SR, AlAmoudi A, et al. 2014. The adaptor ASC has extracellular and "prionoid" activities that propagate inflammation. Nature Immunol 15: 727-737.

Goubau D, Schlee M, Deddouche S, Pruijssers AJ, Zillinger T, Goldeck M, Schuberth C, Van der Veen AG, Fujimura T, Rehwinkel J, et al. 2014. Antiviral immunity via RIG-Imediated recognition of RNA bearing $5^{\prime}$-diphosphates. Nature 514: 372-375.

Hornung V, Ellegast J, Kim S, Brzozka K, Jung A, Kato H, Poeck H, Akira S, Conzelmann KK, Schlee M, et al. 2006. 5'-Triphosphate RNA is the ligand for RIG-I. Science 314: 994-997.

Hou F, Sun L, Zheng H, Skaug B, Jiang QX, Chen ZJ. 2011. MAVS forms functional prion-like aggregates to activate and propagate antiviral innate immune response. Cell 146: $448-461$

Hu Z, Zhou Q, Zhang C, Fan S, Cheng W, Zhao Y, Shao F Wang HW, Sui SF, Chai J. 2015. Structural and biochemical basis for induced self-propagation of NLRC4. Science 350: 399-404.
Iwasaki A, Medzhitov R. 2010. Regulation of adaptive immunity by the innate immune system. Science 327 : 291295.

Jiang X, Kinch LN, Brautigam CA, Chen X, Du F, Grishin NV, Chen ZJ. 2012. Ubiquitin-induced oligomerization of the RNA sensors RIG-I and MDA5 activates antiviral innate immune response. Immunity 36: 959-973.

Jucker M, Walker LC. 2013. Self-propagation of pathogenic protein aggregates in neurodegenerative diseases. Nature 501: 45-51.

Kawai T, Takahashi K, Sato S, Coban C, Kumar H, Kato H, Ishii KJ, Takeuchi O, Akira S. 2005. IPS-1, an adaptor triggering RIG-I- and Mda5-mediated type I interferon induction. Nat Immunol 6: 981-988.

Lamkanfi M, Dixit VM. 2014. Mechanisms and functions of inflammasomes. Cell 157: 1013-1022.

Li XD, Sun L, Seth RB, Pineda G, Chen ZJ. 2005. Hepatitis C virus protease NS3/4A cleaves mitochondrial antiviral signaling protein off the mitochondria to evade innate immunity. Proc Natl Acad Sci 102: 17717-17722.

Li J, McQuade T, Siemer AB, Napetschnig J, Moriwaki K, Hsiao YS, Damko E, Moquin D, Walz T, McDermott A, et al. 2012. The RIP1/RIP3 necrosome forms a functional amyloid signaling complex required for programmed necrosis. Cell 150: 339-350.

Liepinsh E, Barbals R, Dahl E, Sharipo A, Staub E, Otting G. 2003. The death-domain fold of the ASC PYRIN domain presenting a basis for PYRIN/PYRIN recognition. J Mol Biol 332: 1155-1163.

Liu S, Chen J, Cai X, Wu J, Chen X, Wu YT, Sun L, Chen ZJ. 2013. MAVS recruits multiple ubiquitin E3 ligases to activate antiviral signaling cascades. eLife 2: e00785.

Liu S, Cai X, Wu J, Cong Q, Chen X, Li T, Du F, Ren J, Wu YT, Grishin NV, et al. 2015. Phosphorylation of innate immune adaptor proteins MAVS, STING, and TRIF induces IRF3 activation. Science 347: aaa2630.

Lu A, Magupalli VG, Ruan J, Yin Q, Atianand MK, Vos MR, Schröder GF, Fitzgerald KA, Wu H, Egelman EH. 2014. Unified polymerization mechanism for the assembly of ASC-dependent inflammasomes. Cell 156: 1193-1206.

Martinon F, Burns K, Tschopp J. 2002. The inflammasome: A molecular platform triggering activation of inflammatory caspases and processing of proIL- $\beta$. Mol Cell 10: 417-426.

Mathur V, Seuring C, Riek R, Saupe SJ, Liebman SW. 2012. Localization of HET-S to the cell periphery, not to [Het-s] aggregates, is associated with [Het-s]HET-S toxicity. Mol Cell Biol 32: 139-153.

Meylan E, Curran J, Hofmann K, Moradpour D, Binder M, Bartenschlager R, Tschopp J. 2005. Cardif is an adaptor protein in the RIG-I antiviral pathway and is targeted by hepatitis C virus. Nature 437: 1167-1172.

Morrone SR, Matyszewski M, Yu X, Delannoy M, Egelman EH, Sohn J. 2015. Assembly-driven activation of the AIM2 foreign-dsDNA sensor provides a polymerization template for downstream ASC. Nat Commun 6: 7827.

Park HH, Lo YC, Lin SC, Wang L, Yang JK, Wu H. 2007. The death domain superfamily in intracellular signaling of apoptosis and inflammation. Annu Rev Immunol 25: 561-586. 
X. Cai et al.

Peisley A, Wu B, Xu H, Chen ZJ, Hur S. 2014. Structural basis for ubiquitin-mediated antiviral signal activation by RIG-I. Nature 509: 110-114.

Potter JA, Randall RE, Taylor GL. 2008. Crystal structure of human IPS-1/MAVS/VISA/Cardif caspase activation recruitment domain. BMC Struct Biol 8: 11.

Prusiner SB. 1982. Novel proteinaceous infectious particles cause scrapie. Science 216: 136-144.

Ramaswami M, Taylor JP, Parker R. 2013. Altered ribostasis: RNA-protein granules in degenerative disorders. Cell 154: 727-736.

* Riek R, Saupe SJ. 2016. The HET-S/s prion motif in the control of programmed cell death. Cold Spring Harb Perspect Biol doi: 10.1101/cshperspect.a023515.

Saupe SJ. 2011. The [Het-s] prion of Podospora anserina and its role in heterokaryon incompatibility. Semin Cell Dev Biol 22: 460-468.

Seth RB, Sun L, Ea CK, Chen ZJ. 2005. Identification and characterization of MAVS, a mitochondrial antiviral signaling protein that activates NF-кB and IRF 3. Cell 122: 669-682.

Seuring C, Greenwald J, Wasmer C, Wepf R, Saupe SJ, Meier BH, Riek R. 2012. The mechanism of toxicity in HET-S/ HET-s prion incompatibility. PLoS Biol 10: e1001451.

Shi Y, Yuan B, Qi N, Zhu W, Su J, Li X, Qi P, Zhang D, Hou F. 2015. An autoinhibitory mechanism modulates MAVS activity in antiviral innate immune response. Nat Commun 6: 7811

Su L, Quade B, Wang H, Sun L, Wang X, Rizo J. 2014. A plug release mechanism for membrane permeation by MLKL. Structure 22: 1489-1500.

Sun Q, Sun L, Liu HH, Chen X, Seth RB, Forman J, Chen ZJ. 2006. The specific and essential role of MAVS in antiviral innate immune responses. Immunity 24: 633-642.

Sun L, Wang H, Wang Z, He S, Chen S, Liao D, Wang L, Yan J, Liu W, Lei X, et al. 2012. Mixed lineage kinase domainlike protein mediates necrosis signaling downstream of RIP3 kinase. Cell 148: 213-227.

Sun L, Wu J, Du F, Chen X, Chen ZJ. 2013. Cyclic GMP-AMP synthase is a cytosolic DNA sensor that activates the type I interferon pathway. Science 339: 786-791.
Takeuchi O, Akira S. 2010. Pattern recognition receptors and inflammation. Cell 140: 805-820.

Tuite MF, Serio TR. 2010. The prion hypothesis: From biological anomaly to basic regulatory mechanism. Nat Rev Mol Cell Biol 11: 823-833.

Wu J, Sun L, Chen X, Du F, Shi H, Chen C, Chen ZJ. 2013. Cyclic GMP-AMP is an endogenous second messenger in innate immune signaling by cytosolic DNA. Science 339: $826-830$.

Wu B, Peisley A, Tetrault D, Li Z, Egelman EH, Magor KE, Walz T, Penczek PA, Hur S. 2014. Molecular imprinting as a signal-activation mechanism of the viral RNA sensor RIG-I. Mol Cell 55: 511-523.

Xu LG, Wang YY, Han KJ, Li LY, Zhai Z, Shu HB. 2005. VISA is an adapter protein required for virus-triggered IFN- $\beta$ signaling. Mol Cell 19: 727-740.

Xu H, He X, Zheng H, Huang LJ, Hou F, Yu Z, de la Cruz MJ, Borkowski B, Zhang X, Chen ZJ, et al. 2014. Structural basis for the prion-like MAVS filaments in antiviral innate immunity. eLife 3: e01489.

Xu H, He X, Zheng H, Huang LJ, Hou F, Yu Z, de la Cruz MJ, Borkowski B, Zhang X, Chen ZJ, et al. 2015. Correction: Structural basis for the prion-like MAVS filaments in antiviral innate immunity. eLife 4: e07546.

Yoneyama M, Fujita T. 2009. RNA recognition and signal transduction by RIG-I-like receptors. Immunol Rev 227: 54-65.

Yoneyama M, Kikuchi M, Natsukawa T, Shinobu N, Imaizumi T, Miyagishi M, Taira K, Akira S, Fujita T. 2004. The RNA helicase RIG-I has an essential function in doublestranded RNA-induced innate antiviral responses. Nat Immunol 5: 730-737.

Zeng W, Xu M, Liu S, Sun L, Chen ZJ. 2009. Key role of Ubc5 and lysine-63 polyubiquitination in viral activation of IRF3. Mol Cell 36: 315-325.

Zeng W, Sun L, Jiang X, Chen X, Hou F, Adhikari A, Xu M, Chen ZJ. 2010. Reconstitution of the RIG-I pathway reveals a signaling role of unanchored polyubiquitin chains in innate immunity. Cell 141: 315-330.

Zou J, Chang M, Nie P, Secombes CJ. 2009. Origin and evolution of the RIG-I like RNA helicase gene family. BMC Evol Biol 9: 85. 


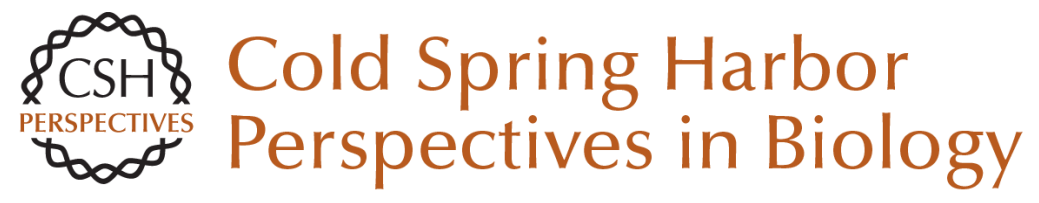

\section{Prion-Like Polymerization in Immunity and Inflammation}

Xin Cai, Hui Xu and Zhijian J. Chen

Cold Spring Harb Perspect Biol 2017; doi: 10.1101/cshperspect.a023580 originally published online November 23, 2016

\section{Subject Collection Prion Biology}

Genetic PrP Prion Diseases

Mee-Ohk Kim, Leonel T. Takada, Katherine Wong, et al.

Neurodegenerative Disease Transmission and Transgenesis in Mice Brittany N. Dugger, Daniel P. Perl and George A. Carlson

Toward the Atomic Structure of PrPSc Jose A. Rodriguez, Lin Jiang and David S. Eisenberg

Bioassays and Inactivation of Prions Kurt Giles, Amanda L. Woerman, David B. Berry, et al.

Functional Prions in the Brain Joseph B. Rayman and Eric R. Kandel

The Amyloid Phenomenon and Its Links with Human Disease Christopher M. Dobson

Tau Positron Emission Tomography Imaging Hartmuth C. Kolb and José Ignacio Andrés

Prion-Like Polymerization in Immunity and Inflammation

Xin Cai, Hui Xu and Zhijian J. Chen
Clinical Neurology and Epidemiology of the Major Neurodegenerative Diseases Michael G. Erkkinen, Mee-Ohk Kim and Michael D. Geschwind

Prion Properties of SOD1 in Amyotrophic Lateral Sclerosis and Potential Therapy Caroline Sibilla and Anne Bertolotti

Mapping Neurodegenerative Disease Onset and Progression William W. Seeley

Erratum: Functional Prions in the Brain Joseph B. Rayman and Eric R. Kandel

Pathology of Neurodegenerative Diseases Brittany N. Dugger and Dennis W. Dickson

TIA-1 Is a Functional Prion-Like Protein Joseph B. Rayman and Eric R. Kandel

Molecular Genetics of Neurodegenerative Dementias

Flora I. Hinz and Daniel H. Geschwind

Cross- $\beta$ Polymerization of Low Complexity

Sequence Domains

Masato Kato and Steven L. McKnight

For additional articles in this collection, see http://cshperspectives.cshlp.org/cgi/collection/

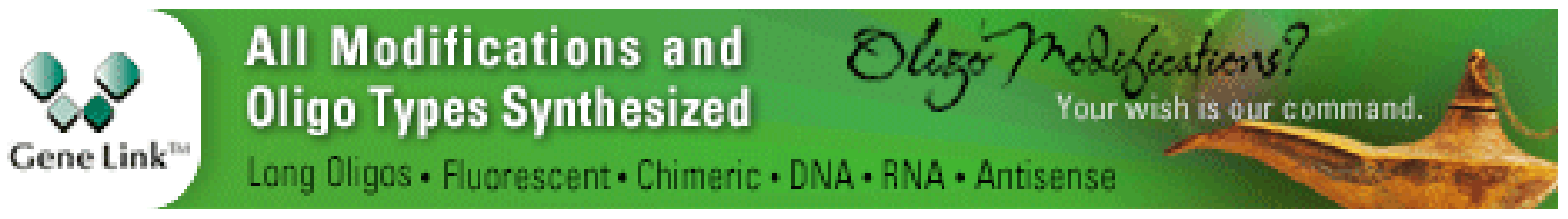

